Philosophical Magazine B, 1999, Vol. 79, No. 10, 1531-1548

\title{
Plasmon excitations in carbon nanotubes
}

\section{Thomas Stöckli† $\|$, Zhong Lin Wang Pierre Stadelmann§̧, and André Châtelain $\dagger$}

† Institut de Physique Expérimentale, Département de Physique, Ecole

Polytechnique Fédérale de Lausanne, CH-1015 Lausanne, Switzerland $\$$ School of Materials Science and Engineering, Georgia Institute of Technology, Atlanta, Georgia 30332-0245, USA

$\S$ Centre Interdépartemental de Microscopie Electronique, Ecole Polytechnique Fédérale de Lausanne, CH-1015 Lausanne, Switzerland

[Received 16 November 1998 and accepted 29 April 1999]

\begin{abstract}
Electron-energy-loss spectroscopy (EELS) in a high-resolution transmission electron microscope can be used to study the excitation of plasmons in nanometre-size particles with high spatial resolution. For isotropic particles of various shapes, models which allow the attribution of the experimental peaks to a certain excitation mechanism and to understand size- or geometry-dependent variations are well established. Recently, locally anisotropic particles such as nested concentric-shell fullerenes and carbon nanotubes have been discovered and have attracted considerable interest. The plasmon losses of these anisotropic particles measured by EELS could contribute to a better understanding of their physical properties, once the theoretical basis for the interpretation is adapted for anisotropic particles. Encouraged by very good qualitative agreement between a model of the plasmons of nested concentricshell fullerenes based on non-relativistic local dielectric response theory with experimental data, we present here a model for the plasmon excitations of multiwall carbon nanotubes based on the same theoretical approach.
\end{abstract}

\section{§1. INTRODUCTION}

Multiwall carbon nanostructures consist of several graphene sheets rolled up into cylinders (Iijima 1991, Ebbesen and Ajayan 1992) or spheres (Ugarte 1992) (figure 1) which are arranged coaxially or concentrically and belong to what are commonly called carbon nanostructures. $\mathrm{C}_{60}$ fullerenes were the first of these structures to be discovered (Kroto et al. 1985). When it became possible to produce them in macroscopic quantities and in crystalline form (Krätscher et al. 1990), it was found that they had remarkable physical properties. Unlike crystalline graphite which is semimetallic, or diamond which is insulating, the $\mathrm{C}_{60}$ crystals were semiconducting with a well defined gap.

When multiwall graphitic tubular structures were discovered (Iijima 1991, Ebbesen and Ajayan 1992) the question about their properties was immediate. At first, simulations of the electronic properties of single-wall tubular structures (singlewall carbon nanotubes) suggested that the electronic properties should strongly

|| Email: thomas.stoeckli@ipe.dp.epfl.ch.

Philosophical Magazine B ISSN 0141-8637 print/ISSN 1463-6417 online (C) 1999 Taylor \& Francis Ltd http://www.tandf.co.uk/JNLS/phb.htm 

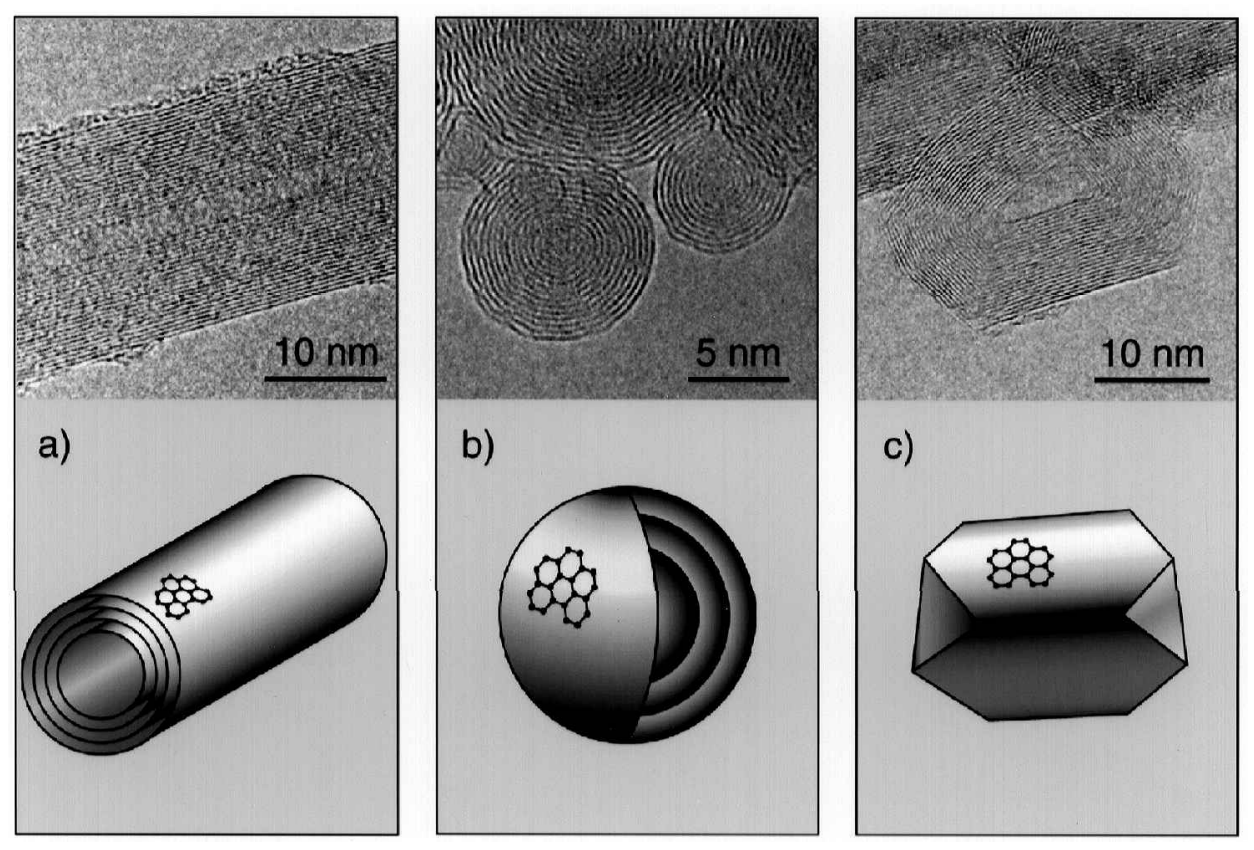

Figure 1. Multiwall carbon nanostructures exist in different geometries: $(a)$ multiwall carbon nanotubes, $(b)$ nested concentric-shell fullerenes; and $(c)$ multiwall polyhedal particles. High-resolution transmission electron micrographs and a schematic of the structure of each type are shown.

depend on the diameter and on the helicity of the tubes (Hamada et al. 1992, Mintmire et al. 1992, Saito et al. 1992). However, the experimental determination of the properties of the different nanostructures and the comparison with the simulations turned out to be a difficult task. Even though important progress has been made, the present production (Ebbesen and Ajayan 1992, Journet et al. 1997, Thess et al. 1996) and purification methods (Bonard et al. 1997b, Dujardin et al. 1998) still yield a mixture of different particles. In bulk measurements the difference between the different constituents of the mixture is washed out and the mean response obtained from such experiments is not suitable for a direct comparison with simulations.

Recently local probe techniques such as field emission microscopy (FEM) (de Heer et al. 1995, Bonard et al. 1997a), scanning tunnelling microscopy (STM) (Carroll et al. 1997, Tans et al. 1997, Bachtold et al. 1998, Bezryadin et al. 1998, Frank et al. 1998), and atomic force microscopy (AFM) (Salvetat et al. 1999) have given some insight into the intriguing properties of those novel carbon-based materials. Using FEM it was possible to show that the tips of nanotubes are characterized by well localized electron states from which the field emission takes place preferentially (de Heer et al. 1995, Bonard et al. 1997a). STM measurements allowed for the first time a direct comparison of the electronic properties of single-wall carbon nanotubes with the calculated density of states (DOS) (Carrol et al. 1997, Tans et al. 1997). Another experimental set-up using STM principally for high-resolution characterization allowed multiprobe transport measurements to be carried out on single-wall carbon nanotubes (Bachtold et al. 1998, Bezryadin et al. 1998). Also 
using the set-up of STM, the conductivity of multiwall carbon nanotubes has been determined. It appears to be quantized, hinting that the transport in these structures may be ballistic even at room temperature (Frank et al. 1998). Finally, AFM measurements revealed that both single-wall and multiwall tubular structures are extremely stiff (Salvetat et al. 1999).

Another technique giving complementary information about the electronic properties of nanometre-size particles is electron-energy-loss spectroscopy (EELS) in a high-resolution transmission electron microscope. Different experimental studies using this technique have been carried out on carbon nanostuctures (Ajayan et al. 1992, Bursill et al. 1992, Kuzuo et al. 1992, Stéphan et al. 1996, Yase et al. 1996, Stöckli et al. 1997a). For the plasmon region, however no detailed interpretation of the experimental data is available. The reason for this is that, even though much theoretical work on carbon nanostructures has already been done, the excitation of plasmons by high-energy electrons has not been addressed in detail except in a recent publication where we have given the basis for the interpretation of electron-energyloss spectra of nested concentric-shell fullerenes (Stöckli et al. 1998a). Using the same approach, based on non-relativistic local dielectric response theory (for a review, see Wang (1996)), we present here the theoretical basis for the interpretation of EELS experiments on multiwall carbon nanotubes, taking into account their particular anisotropy.

Non-relativistic local dielectric response theory has been used with success for the interpretation on isotropic nanometre-size particles of different geometries such as thin slabs (Ritchie 1957), spheres (Ferrel and Echenique 1985, Bausells et al. 1987, Echenique et al. 1987, Ferrell et al. 1987), layered spheres (Ferrell et al. 1987, Ugarte et al. 1992, Stöckli et al. 1997b), spheres halfway embedded in a supporting medium (Wang and Cowley 1987, Zabala and Rivacoba 1991), and cylindrical channels (Chu et al. 1984, Zabala et al. 1989, Walsh 1991, Rivacoba et al. 1995). Since a preliminary comparison of experimental data with the simulations of the anisotropic spheres (nested concentric-shell fullerenes) shows excellent qualitative agreement between theory and measurement (Stöckli et al. 1998b), we are confident that the following results will give a valuable basis for the interpretation of the electron-energy-loss spectra of multiwall carbon nanotubes for penetrating and non-penetrating electrons.

\section{§ 2. NON-RELATIVISTIC LOCAL DIELECTRIC RESPONSE THEORY OF SMALL PARTICLES}

\subsection{General considerations}

The basic idea of non-relativistic local dielectric response theory is to calculate the energy loss of one single probe electron $\dagger$ by integration of the elementary work $\delta \mathrm{W}=\mathbf{F}(\mathbf{x}, \mathrm{t}) \cdot \mathrm{d} \mathbf{x}$ done by the electric field acting on the electron along its trajectory:

$$
\Delta \mathrm{E}=-\int_{\text {trajectory }} \delta \mathrm{W}=\frac{\mathrm{e}}{2 \pi} \int_{\text {trajectory }}\left(\int_{-\infty}^{\infty} \exp (-\mathrm{i} \omega \mathrm{t}) \mathrm{E}(\mathbf{x}, \omega) \mathrm{d} \omega\right) \cdot \mathrm{d} \mathbf{x} .
$$

$\dagger$ In a transmission electron microscope an electron flux of about $10^{12}$ electrons s $^{-1}$ passes through the sample. However, the electrons have a rather high kinetic energy (typically $100 \mathrm{keV}$ and more) and their speed is of the order of half the speed of light. As a consequence, the distance between successive electrons is large enough that the interaction between them can be neglected. 
It is assumed that the electron is travelling on a straight line and at constant velocity $\mathrm{v} \dagger$ so that the evaluation of the path integral is possible.

The electric field needed for the evaluation of the energy loss $\Delta \mathrm{E}$ is determined using the Maxwell equations. Within this formalism, the medium through which the electron is travelling is characterized by its frequency-dependent complex dielectric tensor: . This requires the resolution of the Maxwell equations in frequency space so that the electric field is obtained as frequency-dependent function. However, for the determination of the energy which the electron loses when passing through the sample (equation (1)) the time-dependent electric field needs to be known. In equation (1) the electric field is therefore written as the Fourier transform of the frequency-dependent dielectric response as obtained from the resolution of the Maxwell equations into time space. For the Fourier transform, the following convention has been adapted:

$$
\mathrm{A}(\mathbf{r}, \omega)=\int_{-\infty}^{\infty} \exp (\mathrm{i} \omega \mathrm{t}) \mathrm{A}(\mathbf{r}, \mathrm{t}) \mathrm{dt}
$$

is the direct Fourier transform from time space into frequency space, and

$$
\mathrm{A}(\mathbf{r}, \mathrm{t})=\frac{1}{2 \pi} \int_{-\infty}^{\infty} \exp (-\mathrm{i} \omega \mathrm{t}) \mathrm{A}(\mathbf{r}, \omega) \mathrm{d} \omega
$$

is the inverse transform from frequency into time space.

It has to be noted that the energy lost by the electron upon interaction with the sample can also be expressed in terms of the plasmon excitation probability $\mathrm{dP}(\omega) / \mathrm{d} \omega:$

$$
\Delta \mathrm{E}=\int_{0}^{\infty} \hbar_{\omega} \frac{\mathrm{dP}(\omega)}{\mathrm{d} \omega} \mathrm{d} \omega
$$

Eliminating $\Delta \mathrm{E}$ in equations (1) and (3) the probability $\mathrm{dP}(\omega) / \mathrm{d} \omega$ for an electron to lose the amount of energy $\omega$ can be calculated. This quantity is of considerable interest since it can be compared directly with experimental data.

For the determination of the energy loss (equation (1)) an additional simplification of the original problem is introduced; it is assumed that the probe electron instantaneously reacts with the field which it induces\$. For particles with isotropic electronic properties, the Maxwell equations in this case lead to the Poisson equation

$$
\nabla^{2} \mathbf{V}(\mathbf{r}, \omega)=-\frac{1}{\varepsilon_{0} \varepsilon(\omega)} \rho(\mathbf{r}, \omega) .
$$

For the case when the particle has anisotropic dielectric properties the equation which determines the potential is given by

$\dagger$ The energy loss of the probe electrons in the plasmon region is below $40 \mathrm{eV}$. Compared with the primary energy of more than $100 \mathrm{keV}$, this energy is negligible. Scattering angles of electrons exciting plasmons in the sample are smaller than about $50 \mathrm{mrad}$. In consequence, the trajectory of the probe electrons is in good approximation a straight line.

$\$$ It can be realized that the wave-vector dependence of the dielectric response of the medium is excluded (local response). Even with this simplification, the model has proved successful in explaining the plasmon losses of small particles of various geometries.

$\S$ It is assumed that the terms in $(\mathrm{v} / \mathrm{c})^{2}$ are small compared with the other terms in the Maxwell equations (non-relativistic theory). This means that retardation effects and Cerenkov radiation are neglected. 


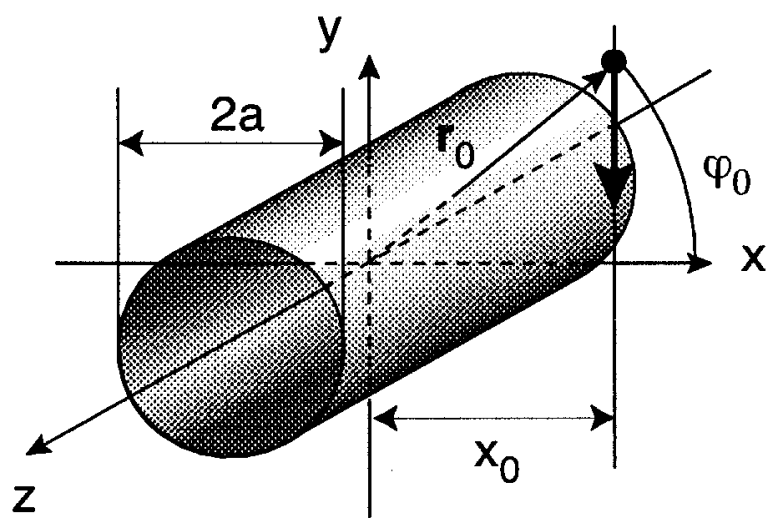

Figure 2. Geometrical definitions for the carbon nanotube geometry. The electron, located at position $\mathbf{r}_{0}$, is travelling at constant velocity at an impact-parameter $x_{0}$ from the origin in negative $y$-direction. In cylindrical coordinates, its position is given by the parameters $r_{0}, \varphi_{0}$ and $z_{0}$.

$$
\nabla \cdot\left[\varepsilon_{0} \tilde{\varepsilon}(\omega) \nabla \mathbf{V}(\mathbf{r}, \omega)\right]=-\rho(\mathbf{r}, \omega) .
$$

$\rho(\mathbf{r}, \omega)$ is the Fourier transform of the point charge located at position $\mathbf{r}$ and, for the geometry shown in figure 2, is given by

$$
\rho(\mathbf{r}, \omega)=\frac{-\mathrm{e}}{\mathrm{v}} \delta\left(\mathrm{x}-\mathrm{x}_{0}\right) \delta(\mathrm{z}) \exp \left(\frac{\mathrm{i} \omega \mathrm{y}}{\mathrm{v}}\right) .
$$

From the viewpoint of classical electrodynamics, the probe electron can interact in two ways with nanometre-size particles. On the one hand it can polarize the medium in which it travels. This polarization requires energy and the corresponding energy loss of the probe electrons can be observed in the electron-energy-loss spectra. The losses due to this excitation mechanism are called volume plasmons. The position of the maxima of the volume plasmon excitation (resonance) are determined by the zeros of the real part of the dielectric function. On the other hand the probe electron can induce surface charges. They enter in resonance at a frequency which is determined by the position of the volume plasmon resonance and the geometry of the particle. The losses due to this excitation mechanism are called surface plasmons and can only be observed when the particles are small enough.

\subsection{Surface plasmon excitation}

Basically, equations (1) and (3) allow the total plasmon excitation probability to be determined using the solution of equations $(4 a)$ and $(4 b)$. However, it is convenient to treat the surface and volume plasmon excitations separately. For this purpose it can be noted that the general solution of equations $(4 a)$ and $(4 b)$ is the sum of the homogeneous and the particular solution of the problem. The two terms represent the induced potential (surface plasmon) and the direct potential (volume plasmon) respectively:

$$
\mathrm{V}(\mathbf{r}, \omega)=\mathrm{V}^{\text {ind }}(\mathbf{r}, \omega)+\mathrm{V}^{\mathrm{p}}(\mathbf{r}, \omega)
$$

Since the induced potential responsible for the surface losses can be calculated separately, it is useful to introduce the notion of surface plasmon excitation prob- 
ability $\mathrm{dP}^{\text {surf }}(\omega) / \mathrm{d} \omega$. Starting from equations (1) and (3) it can be shown that the surface excitation probability for the geometry shown in figure 2 is given by the following expression (Wang 1996) $\dagger$;

$$
\frac{\mathrm{dP}^{\text {surf }}(\omega)}{\mathrm{d} \omega}=\frac{\mathrm{e}}{\pi \hbar_{\mathrm{v}^{2}}^{2}} \int_{-\infty}^{\infty} \mathrm{dy}^{\prime} \int_{-\infty}^{\infty} \mathrm{dy} \operatorname{Im}\left[\left.\exp \left(\frac{\mathrm{i} \omega\left(\mathrm{y}^{\prime}-\mathrm{y}\right)}{\mathrm{v}}\right) \mathrm{v}^{\mathrm{ind}}\left(\mathbf{r}, \mathbf{r}_{0}\right)\right|_{\substack{\mathbf{r}_{0}=\left(\mathrm{x}_{0}, \mathrm{y}^{\prime}, 0\right) \\ \mathbf{r}=\left(\mathrm{x}_{0}, \mathrm{y}, 0\right)}}\right] \text {. }
$$

It is important to note that the time dependence of the problem has been eliminated. $\mathrm{V}^{\text {ind }}\left(\mathbf{r}, \mathbf{r}_{0}\right)$ is the induced potential at position $\mathbf{r}$ caused by a stationary electron located at position $r_{0}$. It is the homogeneous part of the solution of

$$
\nabla^{2} \mathrm{~V}\left(\mathbf{r}, \mathbf{r}_{0}\right)=\frac{\mathrm{e}}{\varepsilon_{0} \varepsilon(\omega)} \delta\left(\mathbf{r}-\mathbf{r}_{0}\right)
$$

if the electron is travelling in an isotropic medium and of

$$
\nabla \cdot\left[\tilde{\varepsilon}(\omega) \nabla \mathrm{V}\left(\mathbf{r}, \mathbf{r}_{0}\right)\right]=\frac{\mathrm{e}}{\varepsilon_{0}} \delta\left(\mathbf{r}-\mathbf{r}_{0}\right)
$$

if it is in an anisotropic medium. The potential distribution therefore is quasi-electrostatic and frequency dependent for each point along the trajectory of the incident electron. The integral over y is the sum over the contributions of all the points along the trajectory.

\subsection{Volume plasmon excitation}

The volume plasmon excitation in a uniaxial crystal such as graphite has been treated theoretically by different workers (Hubbard 1955a, b, Tosatti 1969, Wessjohann 1974, Daniels et al. 1979). Probably the most detailed calculations have been published by Wessjohann (1974). For a uniaxial crystal with its c axis inclined by an angle $\alpha$ with respect to the optical axes of the microscope (figure 3 ) the volume plasmon excitation probability per unit path length is given by

$$
\frac{\mathrm{d}^{2} \mathrm{P}^{\text {volume }}(\omega)}{\mathrm{d} \omega \mathrm{dy}}=\frac{\mathrm{e}^{2}}{4 \pi^{3} \mathrm{v}^{2} \varepsilon_{0} \hbar} \int_{0}^{\theta_{\mathrm{c}}} \theta \mathrm{d} \theta \int_{0}^{2 \pi} \mathrm{d} \varphi \operatorname{Im}\left(\frac{-\mathrm{q}_{0}^{2}}{\mathrm{q}_{\mathrm{p}}^{2} \varepsilon_{\perp}(\omega)+\mathrm{q}_{\mathrm{c}}^{2} \varepsilon_{\|}(\omega)}\right)
$$

$\mathrm{q}_{\mathrm{c}}$ and $\mathrm{q}_{\mathrm{p}}$ are the projection of the transferred momentum $\mathbf{q}$ on to the coordinate system in which the dielectric tensor is diagonal, that is on the unit vector parallel to the $\mathrm{c}$ axis of graphite and on to the plane perpendicular to the $\mathrm{c}$ axis respectively (figure 3). The two projections can be expressed in terms of the scattering angle $\theta$, the azimuthal angle $\varphi$ and the angle $\alpha$ between the $\mathrm{c}$ axis of the crystal and the optical axis (figure 3):

$$
\begin{aligned}
& \mathrm{q}_{\mathrm{p}}^{2}=\mathrm{q}_{0}^{2}\left[\left(\theta_{\mathrm{E}} \sin \alpha-\theta \cos \varphi \cos \alpha\right)^{2}+(\theta \sin \varphi)^{2}\right] \\
& \mathrm{q}_{\mathrm{c}}^{2}=\mathrm{q}_{0}^{2}\left[\theta_{\mathrm{E}} \cos \alpha-\theta \cos \varphi \sin \alpha\right]^{2},
\end{aligned}
$$

where $\theta_{\mathrm{E}}$ is given by

$$
\theta_{\mathrm{E}}=\frac{\omega}{2 \pi \mathrm{vq}_{0}}
$$

$\dagger$ Because in our case the electron is assumed to move in the opposite direction compared with that in review by Wang (1996), the sign of the expression in the imaginary part needs to be plus instead of minus. 


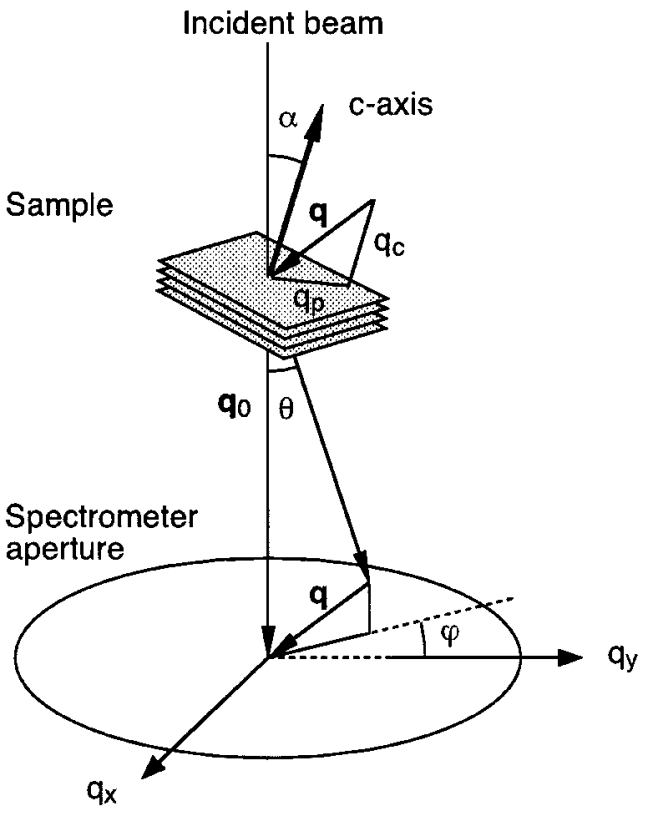

Figure 3. Momentum transfer components for a probe electron passing through a uniaxial crystal. The crystal's $c$ axis is tilted by an angle $\alpha$ with respect to the incident electron beam. Before the scattering event, the electron has a momentum of $\mathbf{q}_{0}$. During the scattering event, it transfers the quantity q to the crystal and leaves deviated by the angle $\theta$ with respect to its incident direction and by the azimuthal angle $\varphi$.

The integration over the angles $\theta$ and $\varphi$ takes into account all electrons scattered within an angle smaller than the cut-off angle $\theta_{\mathrm{c}}$ (Egerton 1996).

\section{§ 3. Modelling THE DieleCtric PROPERTIES OF a CARBON NANOTUBE}

Multiwall carbon nanotubes consist of several graphene sheets rolled up to cylinders of different diameters, embedded coaxially into one another so that the distance between the layers is approximately equal to the interlayer distance of planar graphite (figure 1). In contrast with nested concentric-shell fullerenes, which are almost always completely filled (one can imagine the innermost shell to be a $\mathrm{C}_{60}$ fullerene), carbon nanotubes are hollow. This means not only that surface plasmons exist on the outer surface but also that there are additional surface modes on the inner surface and coupling modes between the surfaces. For mathematical convenience, but also because the plasmons on the inner surface are screened by the body of the nanotube, we don't take into account the inner hollow in the calculations of the surface plasmon excitation probability $\dagger(\S 4)$. Since the volume plasmon contribution is far more important than the surface contribution as long as the tube has more than about five layers, it will, however, be important to correct the volume plasmon excitation probability for the inner hollow (\$5).

$\dagger$ The approach presented in this paper is also suitable for calculating the surface modes on the inner surface. In fact it would be sufficient to include an additional boundary between the tube and the inner hollow. The potential could then be calculated exactly in the same way for the three different regions. 
a)

Planar graphite:

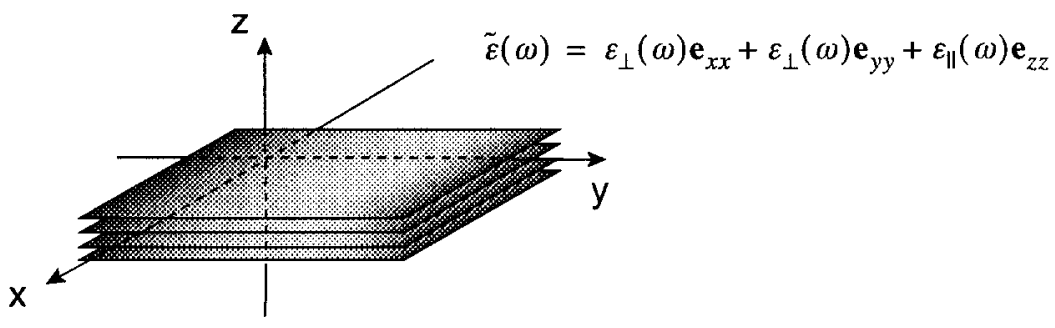

b)

Carbon nanotube:

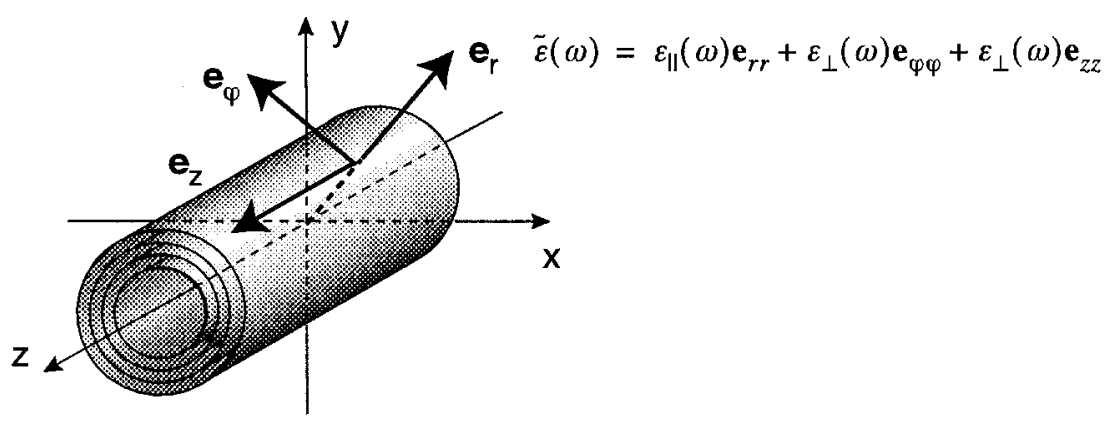

Figure 4. Dielectic tensor for $(a)$ planar graphite and $(b)$ carbon nanotube model. The dielectric tensor of graphite is projected into cylindrical coordinates.

In order to model the dielectric response of a carbon nanotube we follow the procedure of Lucas et al. (1994) which consists in projecting of the dielectric tensor of planar graphite into cylindrical geometry (figure 4). For planar graphite the dielectric tensor has the form of a diagonal matrix with two different components along the natural crystallographic directions (figure $4(a)$ ):

$$
\tilde{\boldsymbol{\varepsilon}}(\omega)=\varepsilon_{\perp}(\omega) \mathbf{e}_{\mathrm{xx}}+\varepsilon_{\perp}(\omega) \mathbf{e}_{\mathrm{yy}}+\varepsilon_{\|}(\omega) \mathbf{e}_{\mathrm{zz}} .
$$

$\varepsilon_{\perp}(\omega)$ describes the dielectric response of graphite for an electric field perpendicular to the $\mathrm{c}$ axis and $\varepsilon_{\|}(\omega)$ is the response for an electric field parallel to the $\mathrm{c}$ axis. From purely geometrical considerations, the projection of the dielectric tensor of planar graphite into cylindrical coordinates is given by

$$
\tilde{\boldsymbol{\varepsilon}}(\omega)=\varepsilon_{\|}(\omega) \mathbf{e}_{\mathrm{rr}}+\varepsilon_{\perp}(\omega) \mathbf{e}_{\varphi \varphi}+\varepsilon_{\perp}(\omega) \mathbf{e}_{\mathrm{zz}} .
$$

\section{§ 4. Determination of the surface excitation probability}

The problem consists in finding the solution of equations (8) and (9) for the geometry shown in figure 2 . The carbon nanotube of infinite length is located with its symmetry axis on the $\mathrm{z}$ axis of a Cartesian coordinate system $(\mathrm{x}, \mathrm{y}, \mathrm{z})$. The probe electron, located at position $\mathbf{r}_{0}$, is parametrized in cylindrical coordinates $r_{0}, \varphi_{0}$ and $z_{0}$. Owing to symmetry, $z_{0}$ can be chosen to be equal to zero. Since it is assumed that the electron is moving on a straight line at an impact parameter $\mathrm{x}_{0}$ from the optical 
axis of the microscope (y axis), $r_{0}$ and $\varphi_{0}$ can be expressed as functions of the impact parameter and $\mathrm{y}: \mathrm{r}_{0}=\left(\mathrm{y}^{2}+\mathrm{x}_{0}^{2}\right)^{1 / 2}$ and $\cos \varphi_{0}=\mathrm{x}_{0} / \mathrm{r}_{0}$.

For the determination of the potential, two cases need to be distinguished, namely the electron located outside and inside the nanotube. The potentials for the two cases are denoted as $\mathrm{V}^{\text {in }}\left(\mathbf{r}, \mathbf{r}_{0}\right)$ and $\mathrm{V}^{\text {out }}\left(\mathbf{r}, \mathbf{r}_{0}\right)$ respectively, where $r$ is an arbitrary position in space and $\mathbf{r}_{0}$ is the electron position (figure 2 ).

\subsection{Potential distribution for an electron outside the cylinder}

The equations that determine the potential distribution for an electron travelling outside the cylinder are

$$
\nabla^{2} \mathrm{~V}_{\mathrm{r}>\mathrm{a}}^{\text {out }}\left(\mathbf{r}, \mathbf{r}_{0}\right)=-\frac{1}{\varepsilon_{0}} \rho\left(\mathbf{r}, \mathbf{r}_{0}\right) \quad \text { for } \quad \mathrm{r}>\mathrm{a}
$$

and

$$
\nabla \cdot\left[\tilde{\varepsilon}(\omega) \nabla \mathrm{V}_{\mathrm{r}<\mathrm{a}}^{\text {out }}\left(\mathbf{r}, \mathbf{r}_{0}\right)\right]=0 \quad \text { for } \quad \mathrm{r}<\mathrm{a} .
$$

$\rho\left(\mathbf{r}, \mathbf{r}_{0}\right)=-\mathrm{e} \delta\left(\mathbf{r}-\mathbf{r}_{0}\right)$ represents an electron located at $\mathbf{r}_{0}$ and $\mathrm{a}$ is the radius of the cylinder (see figure 2 ).

As discussed in $\S 2.2$, the solution of equation $(15 a)$ is written as the sum of the solution of the homogeneous problem, $\mathrm{V}_{\mathbf{r}>\mathrm{a}}^{\text {out ind }}\left(\mathbf{r}, \mathbf{r}_{0}\right)$ and the solution of the inhomogeneous problem, $\mathbf{V}_{\mathrm{r}>\mathrm{a}}^{\text {out }} \mathrm{p}\left(\mathbf{r}, \mathbf{r}_{0}\right)$.

$$
\mathrm{V}_{\mathrm{r}>\mathrm{a}}^{\text {out }}\left(\mathbf{r}, \mathbf{r}_{0}\right)=\mathrm{V}_{\mathrm{r}>\mathrm{a}}^{\text {out ind }}\left(\mathbf{r}, \mathbf{r}_{0}\right)+\mathrm{V}_{\mathrm{r}>\mathrm{a}}^{\text {out }}\left(\mathbf{r}, \mathbf{r}_{0}\right) \text {. }
$$

Equation (15a) with the inhomogeneous term representing a point charge located at $r_{0}$ is frequently encountered in classical electrodynamics and its particular solution is

$$
\mathrm{V}_{\mathrm{r}>\mathrm{a}}^{\text {out }} \mathrm{p}\left(\mathbf{r}, \mathbf{r}_{0}\right)=-\frac{\mathrm{e}}{4 \pi \varepsilon_{0}\left|\mathbf{r}-\mathbf{r}_{0}\right|}
$$

For our problem, this solution is rewritten as a Fourier Bessel expansion (Jackson 1975):

$$
\begin{aligned}
\mathrm{V}_{\mathrm{r}>\mathrm{a}}^{\text {out }} \mathrm{p}\left(\mathbf{r}, \mathbf{r}_{0}\right)= & -\frac{\mathrm{e}}{2 \pi \varepsilon_{0}} \sum_{\mathrm{m} \geqslant 0}\left(2-\delta_{0, \mathrm{~m}}\right) \\
& \times \cos \left[\mathrm{m}\left(\varphi-\varphi_{0}\right)\right] \int_{-\infty}^{\infty} \frac{\mathrm{dq}}{2 \pi} \exp (\text { iqz }) \mathrm{L}_{\mathrm{m}}\left(|\mathrm{q}| \mathrm{r},|\mathrm{q}| \mathrm{r}_{0}\right),
\end{aligned}
$$

where

$$
\mathrm{L}_{\mathrm{m}}\left(|\mathrm{q}| \mathrm{r},|\mathrm{q}| \mathrm{r}_{0}\right)=\mathrm{K}_{\mathrm{m}}(|\mathrm{q}| \mathrm{r}) \mathrm{I}_{\mathrm{m}}\left(|\mathrm{q}| \mathrm{r}_{0}\right) \theta\left(\mathrm{r}-\mathrm{r}_{0}\right)+\mathrm{K}_{\mathrm{m}}\left(|\mathrm{q}| \mathrm{r}_{0}\right) \mathrm{I}_{\mathrm{m}}(|\mathrm{q}| \mathrm{r}) \theta\left(\mathrm{r}_{0}-\mathrm{r}\right) .
$$

In equation $(18 b), \theta(\mathrm{x})$ is the Heaviside step function, defined as

$$
\theta(\mathrm{x})=\left\{\begin{array}{lll}
1 & \text { for } & \mathrm{x}>0 \\
0 & \text { for } & \mathrm{x}<0
\end{array}\right.
$$

and $\mathrm{K}_{\mathrm{m}}(|\mathrm{q}| \mathrm{r})$ and $\mathrm{I}_{\mathrm{m}}(|\mathrm{q}| \mathrm{r})$ are the modified Bessel functions (Bessel function of purely imaginary argument (Watson 1996)) of order $m$ and of argument $|\mathrm{q}| \mathrm{r}$.

The homogeneous solution of equation $(15 a)$ is also written as a Fourier Bessel expansion with coefficients $\mathrm{A}_{\mathrm{m}}(\mathrm{q}, \omega)$ that are determined by the boundary conditions: 


$$
\begin{aligned}
\mathrm{V}_{\mathrm{r}>\mathrm{a}}^{\text {out ind }}\left(\mathbf{r}, \mathbf{r}_{0}\right)= & -\frac{\mathrm{e}}{2 \pi \varepsilon_{0}} \sum_{\mathrm{m} \geqslant 0}\left(2-\delta_{0, \mathrm{~m}}\right) \\
& \times \cos \left[\mathrm{m}\left(\varphi-\varphi_{0}\right)\right] \int_{-\infty}^{\infty} \frac{\mathrm{dq}}{2 \pi} \mathrm{A}_{\mathrm{m}}(\mathrm{q}, \omega) \exp (\text { iqz }) \mathrm{K}_{\mathrm{m}}(|\mathrm{q}| \mathrm{r})
\end{aligned}
$$

The solution of the homogeneous equation $(15 b)$ describing the potential in the anisotropic medium can also be written in the form of a Fourier Bessel expansion similar to equation $(18 a)$. In fact, in cylindrical coordinates, the radial equation is found to be

$$
(\mathrm{kr})^{2} \frac{\mathrm{d}}{\mathrm{d}(\mathrm{kr})^{2}} \mathrm{~V}(\mathrm{kr})+\mathrm{kr} \frac{\mathrm{d}}{\mathrm{d}(\mathrm{kr})} \mathrm{V}(\mathrm{kr})-\left((\mathrm{kr})^{2}+\mathrm{m}^{2} \frac{\varepsilon_{\perp}(\omega)}{\varepsilon_{\|}(\omega)}\right) \mathrm{V}(\mathrm{kr})=0
$$

where $\mathrm{k}=\mathrm{q}\left[\varepsilon_{\perp}(\omega) / \varepsilon_{\|}(\omega)\right]^{1 / 2}$. If the effective azimuthal quantum number $\nu_{\mathrm{m}}(\omega)$ defined by

$$
\nu_{\mathrm{m}}(\omega)=\mathrm{m}\left(\frac{\varepsilon_{\perp}(\omega)}{\varepsilon_{\|}(\omega)}\right)^{1 / 2}
$$

is introduced, equation (21) becomes identical with the radial equation in the homogeneous case. The solution of the homogeneous equation in the anisotropic case can therefore be obtained from the solution of the isotropic case (equation (20)) by replacing the quantum number $\mathrm{m}$ in equation $(20)$ by $\nu_{\mathrm{m}}(\omega)$, so that $\mathrm{V}_{\mathrm{r}<\mathrm{a}}^{\text {out ind }}\left(\mathbf{r}, \mathbf{r}_{0}\right)$ becomes

$$
\begin{aligned}
\mathrm{V}_{\mathrm{r}<\mathrm{a}}^{\text {out ind }}\left(\mathbf{r}, \mathbf{r}_{0}\right) & =-\frac{\mathrm{e}}{2 \pi \varepsilon_{0}} \sum_{\mathrm{m} \geqslant 0}\left(2-\delta_{0, \mathrm{~m}}\right) \\
& \times \cos \left[\mathrm{m}\left(\varphi-\varphi_{0}\right)\right] \int_{-\infty}^{\infty} \frac{\mathrm{dq}}{2 \pi} \mathrm{B}_{\mathrm{m}}(\mathrm{q}, \omega) \exp (\text { iqz }) \mathrm{I}_{\nu_{\mathrm{m}}(\omega)}\left[\left(\frac{\varepsilon_{\perp}(\omega)}{\varepsilon_{\|}(\omega)}\right)^{1 / 2}|\mathrm{q}| \mathrm{r}\right]
\end{aligned}
$$

As in equation (20), the coefficients $B_{m}(q, \omega)$ are unknown and are determined by the boundary conditions.

The boundary conditions, namely that the potential and that the normal component of the displacement field must be continuous,

$$
\left.\mathrm{V}_{\mathrm{r}>\mathrm{a}}^{\text {out }}\left(\mathbf{r}, \mathbf{r}_{0}\right)\right|_{\mathrm{r}=\mathrm{a}}=\left.\mathrm{V}_{\mathrm{r}<\mathrm{a}}^{\text {out }}\left(\mathbf{r}, \mathbf{r}_{0}\right)\right|_{\mathrm{r}=\mathrm{a}}
$$

and

$$
\left.\frac{\mathrm{dV}_{\mathrm{r}>\mathrm{a}}^{\text {out }}\left(\mathbf{r}, \mathbf{r}_{0}\right)}{\mathrm{dr}}\right|_{\mathrm{r}=\mathrm{a}}=\left.\varepsilon_{\|}(\omega) \frac{\mathrm{dV}_{\mathrm{r} \mathbf{a} \mathrm{a}}^{\text {out }}\left(\mathbf{r}, \mathbf{r}_{0}\right)}{\mathrm{dr}}\right|_{\mathrm{r}=\mathrm{a}},
$$

lead to the following expression for the coefficients $A_{m}(q, \omega)$ and $B_{m}(q, \omega)$ :

$$
\begin{aligned}
& \mathrm{A}_{\mathrm{m}}(\mathrm{q}, \omega)=\frac{\mathrm{K}_{\mathrm{m}}\left(|\mathrm{q}| \mathrm{r}_{0}\right)}{\Delta_{\mathrm{m}}(\mathrm{q}, \omega)}\left\{\mathrm{I}_{\mathrm{m}}^{\prime}(|\mathrm{q}| \mathrm{a}) \mathrm{I}_{\nu_{\mathrm{m}}(\omega)}\left[\left(\frac{\varepsilon_{\perp}(\omega)}{\varepsilon_{\|}(\omega)}\right)^{1 / 2}|\mathrm{q}| \mathrm{a}\right]\right. \\
& \left.-\left[\varepsilon_{\perp}(\omega) \varepsilon_{\|}(\omega)\right]^{1 / 2} \mathrm{I}_{\mathrm{m}}(|\mathbf{q}| \mathrm{a}) \mathbf{I}_{\nu_{\mathrm{m}}(\omega)}^{\prime}\left[\left(\frac{\varepsilon_{\perp}(\omega)}{\varepsilon_{\|}(\omega)}\right)^{1 / 2}|\mathrm{q}| \mathrm{a}\right]\right\} \text {, }
\end{aligned}
$$


where

$$
\mathbf{B}_{\mathrm{m}}(\mathbf{q}, \omega)=\frac{\mathrm{K}_{\mathrm{m}}\left(|\mathbf{q}| \mathrm{r}_{0}\right)}{\Delta_{\mathrm{m}}(\mathbf{q}, \omega)}\left(\mathrm{I}_{\mathrm{m}}^{\prime}(|\mathbf{q}| \mathrm{a}) \mathrm{K}_{\mathrm{m}}(|\mathrm{q}| \mathrm{a})-\mathrm{I}_{\mathrm{m}}(|\mathrm{q}| \mathrm{a}) \mathrm{K}_{\mathrm{m}}^{\prime}(|\mathbf{q}| \mathrm{a})\right),
$$

$$
\begin{aligned}
\Delta_{\mathrm{m}}(\mathrm{q}, \omega)= & {\left[\varepsilon_{\perp}(\omega) \varepsilon_{\|}(\omega)\right]^{1 / 2} \mathrm{I}_{\nu_{\mathrm{m}}(\omega)}^{\prime}\left[\left(\frac{\varepsilon_{\perp}(\omega)}{\varepsilon_{\|}(\omega)}\right)^{1 / 2}|\mathrm{q}| \mathrm{a}\right] \mathrm{K}_{\mathrm{m}}(|\mathrm{q}| \mathrm{a}) } \\
& -\mathrm{I}_{\nu_{\mathrm{m}}(\omega)}\left[\left(\frac{\varepsilon_{\perp}(\omega)}{\varepsilon_{\|}(\omega)}\right)^{1 / 2}|\mathrm{q}| \mathrm{a}\right] \mathrm{K}_{\mathrm{m}}^{\prime}(|\mathrm{q}| \mathrm{a}) .
\end{aligned}
$$

As a general convention we use the prime to denote the derivative with respect to the argument of the primed function.

\subsection{Potential distribution for an electron inside the cylinder}

The equations that govern the potential distribution when the electron is travelling inside the cylinder are

$$
\nabla^{2} \mathrm{~V}_{\mathrm{r}>\mathrm{a}}^{\text {in }}\left(\mathbf{r}, \mathbf{r}_{0}\right)=0 \quad \text { for } \mathrm{r}>\mathrm{a}
$$

and

$$
\nabla \cdot\left[\tilde{\varepsilon}(\omega) \nabla \mathrm{V}_{\mathrm{r}<\mathrm{a}}^{\text {in }}\left(\mathbf{r}, \mathbf{r}_{0}\right)\right]=-\frac{1}{\varepsilon_{0}} \rho\left(\mathbf{r}, \mathbf{r}_{0}\right) \quad \text { for } \mathrm{r}<\mathrm{a} .
$$

The homogeneous solution of equations $(27 a)$ and $(27 b)$ can be written in terms of a Fourier Bessel series with coefficients $\mathrm{C}_{\mathrm{m}}(\mathrm{q}, \omega)$ and $\mathrm{D}_{\mathrm{m}}(\mathrm{q}, \omega)$ that are determined by the boundary conditions:

$$
\begin{aligned}
\mathrm{V}_{\mathrm{r}>\mathrm{a}}^{\text {in ind }}\left(\mathbf{r}, \mathbf{r}_{0}\right)= & -\frac{\mathrm{e}}{2 \pi \varepsilon_{0}} \sum_{\mathrm{m} \geqslant 0}\left(2-\delta_{0, \mathrm{~m}}\right) \\
& \times \cos \left[\mathrm{m}\left(\varphi-\varphi_{0}\right)\right] \int_{-\infty}^{\infty} \frac{\mathrm{dq}}{2 \pi} \mathrm{C}_{\mathrm{m}}(\mathrm{q}, \omega) \exp (\text { iqz }) \mathrm{K}_{\mathrm{m}}(|\mathrm{q}| \mathrm{r})
\end{aligned}
$$

and

$$
\begin{aligned}
\mathrm{V}_{\mathrm{r}<\mathrm{a}}^{\text {in ind }}\left(\mathbf{r}, \mathbf{r}_{0}\right)= & -\frac{\mathrm{e}}{2 \pi \varepsilon_{0}} \sum_{\mathrm{m} \geqslant 0}\left(2-\delta_{0, \mathrm{~m}}\right) \\
& \times \cos \left[\mathrm{m}\left(\varphi-\varphi_{0}\right)\right] \int_{-\infty}^{\infty} \frac{\mathrm{dq}}{2 \pi} \mathrm{D}_{\mathrm{m}}(\mathrm{q}, \omega) \exp (\text { iqz }) \mathrm{I}_{\nu_{\mathrm{m}}(\omega)}\left[\left(\frac{\varepsilon_{\perp}(\omega)}{\varepsilon_{\|}(\omega)}\right)^{1 / 2}|\mathrm{q}| \mathrm{r}\right] .
\end{aligned}
$$

For the determination of the coefficients $\mathrm{C}_{\mathrm{m}}(\mathrm{q}, \omega)$ and $\mathrm{D}_{\mathrm{m}}(\mathrm{q}, \omega)$ using the boundary conditions $(24 a)$ and $(24 b)$ it would now be necessary to determine the potential $\mathrm{V}_{\mathrm{r}<\mathrm{a}}^{\text {out }} \mathrm{r}\left(\mathbf{r}, \mathrm{r}_{0}\right)$ created by a point charge inside the nanotube. However, at this point, it can be noted that for the determination of the surface plasmon excitation probability (equation (7)) the direct potential does not need to be known explicitly. In fact, the only reason for calculating $\mathrm{V}_{\mathrm{r}<\mathrm{a}}^{\text {in }}\left(\mathbf{r}, \mathbf{r}_{0}\right)$ resides in the necessity to determine the coefficients $\mathrm{C}_{\mathrm{m}}(\mathrm{q}, \omega)$ and $\mathrm{D}_{\mathrm{m}}(\mathrm{q}, \omega)$ via the boundary conditions $(24 a)$ and $(24 b)$ on the potential. However, these can be replaced by the equivalent boundary conditions on 
the electric field, which in term can be calculated in straight forward manner (see appendix A):

$$
\begin{aligned}
& \left.\mathrm{E}_{\mathrm{r}<\mathrm{a}}^{\mathrm{in}}\left(\mathbf{r}, \mathbf{r}_{0}\right) \cdot \mathbf{e}_{\mathrm{z}}\right|_{\mathrm{r}=\mathrm{a}}=\left.\mathrm{E}_{\mathrm{r}>\mathrm{a}}^{\mathrm{in}}\left(\mathbf{r}, \mathbf{r}_{0}\right) \cdot \mathbf{e}_{\mathrm{z}}\right|_{\mathrm{r}=\mathrm{a}}, \\
& \left.\mathrm{E}_{\mathrm{r}<\mathrm{a}}^{\text {in }}\left(\mathbf{r}, \mathbf{r}_{0}\right) \cdot \mathbf{e}_{\varphi}\right|_{\mathrm{r}=\mathrm{a}}=\left.\mathrm{E}_{\mathrm{r}>\mathrm{a}}^{\text {in }}\left(\mathbf{r}, \mathbf{r}_{0}\right) \cdot \mathbf{e}_{\varphi}\right|_{\mathrm{r}=\mathrm{a}},
\end{aligned}
$$

and

$$
\left.\varepsilon_{\|}(\omega)\left(\mathbf{E}_{\mathrm{r}<\mathrm{a}}^{\mathrm{in}}\left(\mathbf{r}, \mathbf{r}_{0}\right) \cdot \mathbf{e}_{\mathrm{r}}\right)\right|_{\mathrm{r}=\mathrm{a}}=\left.\mathbf{E}_{\mathrm{r}>\mathrm{a}}^{\mathrm{in}}\left(\mathbf{r}, \mathbf{r}_{0}\right) \cdot \mathbf{e}_{\mathrm{r}}\right|_{\mathrm{r}=\mathrm{a}} .
$$

$E_{r \gtrless a}^{\text {in }}\left(r, r_{0}\right) \cdot e_{z}, E_{r \gtrless a}^{\text {in }}\left(r, r_{0}\right) \cdot e_{\varphi}$ and $E_{r \gtrless a}^{\text {in }}\left(r, r_{0}\right) \cdot e_{r}$ are the components in cylindrical coordinates of the electric field inside and outside the particle respectively. Equations $(30 a)$ and $(30 b)$ represent the continuity of the tangential component of the electric field and equation $(30 c)$ the continuity of the normal component of the displacement vector.

In terms of the electric field, the solutions of equations $(27 a)$ and $(27 b)$ are

$$
E_{r>a}^{\text {in }}\left(\mathbf{r}, \mathbf{r}_{0}\right)=-\nabla \mathrm{V}_{\mathrm{r}>\mathrm{a}}^{\text {in }}\left(\mathbf{r}, \mathbf{r}_{0}\right)
$$

and

$$
\begin{aligned}
\mathrm{E}_{\mathrm{r}<\mathrm{a}}^{\mathrm{in}}\left(\mathbf{r}, \mathbf{r}_{0}\right) & =-\nabla \mathrm{V}_{\mathrm{r}<\mathrm{a}}^{\text {in }}\left(\mathbf{r}, \mathbf{r}_{0}\right) \\
& =-\nabla \mathrm{V}_{\mathrm{r}<\mathrm{a}}^{\text {in }}\left(\mathbf{r}, \mathbf{r}_{0}\right)-\nabla \mathrm{V}_{\mathrm{r}<\mathrm{a}}^{\text {in ind }}\left(\mathbf{r}, \mathbf{r}_{0}\right) \\
& =\mathbf{E}_{\mathrm{r}<\mathrm{a}}^{\text {in }}\left(\mathbf{r}, \mathbf{r}_{0}\right)-\nabla \mathrm{V}_{\mathbf{r}<\mathrm{a}}^{\text {in ind }}\left(\mathbf{r}, \mathbf{r}_{0}\right),
\end{aligned}
$$

respectively. $-\nabla V_{r<a}^{\text {in }}\left(r, r_{0}\right)$ has been replaced by $E_{r<a}^{\text {in }}\left(r, r_{0}\right)$ which is given for $r>r_{0}$ by (see appendix A)

$$
\begin{aligned}
\mathrm{E}_{\mathrm{r}<\mathrm{a}}^{\mathrm{in}}\left(\mathbf{r}, \mathbf{r}_{0}\right)= & \frac{\mathrm{e}}{2 \pi \varepsilon_{0}} \sum_{\mathrm{m} \geqslant 0}\left(2-\delta_{0, \mathrm{~m}}\right) \\
& \times \int_{-\infty}^{\infty} \frac{\mathrm{dq}}{2 \pi}\left(\frac{1}{\varepsilon_{\|}(\omega)}|\mathrm{q}| \exp (\mathrm{iqz}) \cos \left[\mathrm{m}\left(\varphi-\varphi_{0}\right)\right] \mathrm{I}_{\mathrm{m}}\left(|\mathrm{q}| \mathrm{r}_{0}\right) \mathrm{K}_{\mathrm{m}}^{\prime}(|\mathrm{q}| \mathrm{r}) \mathbf{e}_{\mathrm{r}}\right. \\
& -\frac{1}{\varepsilon_{\perp}(\omega)} \frac{\mathrm{m} \sin \left[\mathrm{m}\left(\varphi-\varphi_{0}\right)\right]}{\mathrm{r}} \exp (\mathrm{iqz}) \mathrm{I}_{\mathrm{m}}\left(|\mathrm{q}| \mathrm{r}_{0}\right) \mathrm{K}_{\mathrm{m}}(|\mathrm{q}| \mathrm{r}) \mathbf{e}_{\varphi} \\
& \left.+\frac{1}{\varepsilon_{\perp}(\omega)} \mathrm{iq} \exp (\mathrm{iqz}) \cos \left[\mathrm{m}\left(\varphi-\varphi_{0}\right)\right] \mathrm{I}_{\mathrm{m}}\left(|\mathrm{q}| \mathrm{r}_{0}\right) \mathrm{K}_{\mathrm{m}}(|\mathrm{q}| \mathrm{r}) \mathbf{e}_{\mathrm{z}}\right)
\end{aligned}
$$

Using the boundary conditions on the electric and the displacement field, the coefficients $\mathrm{C}_{\mathrm{m}}(\mathrm{q}, \omega)$ and $\mathrm{D}_{\mathrm{m}}(\mathrm{q}, \omega)$ can now be determined:

$$
\begin{aligned}
\mathrm{C}_{\mathrm{m}}(\mathrm{q}, \omega)= & \frac{\mathrm{I}_{\mathrm{m}}\left(|\mathrm{q}| \mathrm{r}_{0}\right)}{\varepsilon_{\perp}(\omega) \Delta_{\mathrm{m}}(\mathrm{q}, \omega)}\left\{\left[\varepsilon_{\perp}(\omega) \varepsilon_{\|}(\omega)\right]^{1 / 2} \mathrm{I}_{\nu_{\mathrm{m}}(\omega)}\left[\left(\frac{\varepsilon_{\perp}(\omega)}{\varepsilon_{\|}(\omega)}\right)^{1 / 2}|\mathrm{q}| \mathrm{a}\right] \mathrm{K}_{\mathrm{m}}(|\mathrm{q}| \mathrm{a})\right. \\
& \left.-\varepsilon_{\perp}(\omega) \mathrm{I}_{\nu_{\mathrm{m}}(\omega)}\left[\left(\frac{\varepsilon_{\perp}(\omega)}{\varepsilon_{\|}(\omega)}\right)^{1 / 2}|\mathrm{q}| \mathrm{a}\right] \mathrm{K}_{\mathrm{m}}^{\prime}(|\mathrm{q}| \mathrm{a})\right\}, \\
\mathrm{D}_{\mathrm{m}}(\mathrm{q}, \omega) & =\frac{\mathrm{I}_{\mathrm{m}}\left(|\mathrm{q}| \mathrm{r}_{0}\right)}{\varepsilon_{\perp}(\omega) \Delta_{\mathrm{m}}(\mathrm{q}, \omega)}\left(\mathrm{K}_{\mathrm{m}}^{\prime}(|\mathrm{q}| \mathrm{a}) \mathrm{K}_{\mathrm{m}}(|\mathrm{q}| \mathrm{a})-\varepsilon_{\perp}(\omega) \mathrm{K}_{\mathrm{m}}(|\mathrm{q}| \mathrm{a}) \mathrm{K}_{\mathrm{m}}^{\prime}(|\mathrm{q}| \mathrm{a})\right) .
\end{aligned}
$$




\subsection{Determination of the surface plasmon excitation probability}

In the preceding section, the potential has been calculated for any possible configuration. Now, the excitation probability (equation (7)) needs to be evaluated. Taking into account the different expressions for the potential, the following expression is obtained:

$$
\begin{aligned}
& \frac{\mathrm{dP}^{\text {surf }}(\omega)}{\mathrm{d} \omega}=\frac{\mathrm{e}}{\pi \hbar_{\mathrm{v}}{ }^{2}} \operatorname{Im}\left(\int _ { - \infty } ^ { - \mathrm { y } _ { 0 } } \mathrm { dy } \operatorname { e x p } ( - \frac { \mathrm { i } \omega \mathrm { y } } { \mathrm { v } } ) \left[\int_{-\infty}^{-\mathrm{y}_{0}} \mathrm{dy} \mathrm{y}^{\prime} \exp \left(\frac{\mathrm{i} \omega \mathrm{y}^{\prime}}{\mathrm{v}}\right) \mathrm{V}_{\mathrm{r}>\mathrm{a}}^{\text {out ind }}\left(\mathbf{r}, \mathbf{r}_{0}\right)\right.\right. \\
& \left.+\int_{-y_{0}}^{y_{0}} d y^{\prime} \exp \left(\frac{i \omega y^{\prime}}{v}\right) V_{r>a}^{\text {in ind }}\left(r, r_{0}\right)+\int_{y_{0}}^{\infty} d y^{\prime} \exp \left(\frac{i \omega y^{\prime}}{v}\right) V_{r>a}^{\text {out ind }}\left(r, r_{0}\right)\right] \\
& +\int_{-y_{0}}^{y_{0}} d y \exp \left(-\frac{i \omega y}{v}\right)\left[\int_{-\infty}^{-y_{0}} d y^{\prime} \exp \left(\frac{i \omega y^{\prime}}{v}\right) V_{r<a}^{\text {out ind }}\left(r, r_{0}\right)\right. \\
& \left.+\int_{-\mathrm{y}_{0}}^{\mathrm{y}_{0}} d \mathrm{y}^{\prime} \exp \left(\frac{\mathrm{i} \omega \mathrm{y}^{\prime}}{\mathrm{v}}\right) \mathrm{V}_{\mathrm{r}<\mathrm{a}}^{\text {in, ind }}\left(\mathbf{r}, \mathbf{r}_{0}\right)+\int_{\mathrm{y}_{0}}^{\infty} d \mathrm{y}^{\prime} \exp \left(\frac{\mathrm{i} \omega \mathrm{y}^{\prime}}{\mathrm{v}}\right) \mathrm{V}_{\mathrm{r}<\mathrm{a}}^{\text {out ind }}\left(\mathbf{r}, \mathbf{r}_{0}\right)\right] \\
& +\int_{\mathrm{y}_{0}}^{\infty} \mathrm{dy} \exp \left(\frac{-\mathrm{i} \omega \mathrm{y}}{\mathrm{v}}\right)\left[\int_{-\infty}^{-\mathrm{y}_{0}} \mathrm{dy^{ \prime }} \exp \left(\frac{\mathrm{i} \omega \mathrm{y}^{\prime}}{\mathrm{v}}\right) \mathrm{V}_{\mathrm{r}>\mathrm{a}}^{\text {out ind }}\left(\mathbf{r}, \mathbf{r}_{0}\right)\right. \\
& +\int_{-y_{0}}^{y_{0}} d y^{\prime} \exp \left(\frac{i \omega y^{\prime}}{v}\right) V_{r>a}^{\text {in ind }}\left(r, r_{0}\right) \\
& \left.\left.\left.+\int_{\mathrm{y}_{0}}^{\infty} d \mathrm{y}^{\prime} \exp \left(\frac{\mathrm{i} \omega \mathrm{y}^{\prime}}{\mathrm{v}}\right) \mathrm{V}_{\mathrm{r}>\mathrm{a}}^{\text {out, ind }}\left(\mathbf{r}, \mathrm{r}_{0}\right)\right]\right\}\left.\right|_{\substack{\mathrm{r}_{0}=\left(\mathrm{x}_{0}, \mathrm{y}^{\prime}, 0\right) \\
\mathrm{r}_{\mathrm{r}=\left(\mathrm{x}_{0}, \mathrm{y}, 0\right)}}}\right) \text {. }
\end{aligned}
$$

The integration boundary $\mathrm{y}_{0}$ is determined by the intersection of the tube circumference with the electron trajectory and is given by $\mathrm{y}_{0}=\left(\mathrm{a}^{2}-\mathrm{x}_{0}^{2}\right)^{1 / 2}$.

By inspection of the different integrals, it is found that equation (34) can be considerably simplified. In fact the double integrals over dy and $\mathrm{dy}^{\prime}$ can be factored out so that only the following one-dimensional integrals remain.

$$
\begin{aligned}
& \mathrm{C}_{\mathrm{m}}^{\mathrm{o}}(\mathrm{q}, \omega)=\int_{\mathrm{y}_{0}}^{\infty} \cos \left(\frac{\omega \mathrm{y}}{\mathrm{v}}\right) \mathrm{K}_{\mathrm{m}}(|\mathrm{q}| \mathrm{r}) \cos (\mathrm{m} \varphi) \mathrm{dy} \\
& \mathrm{C}_{\mathrm{m}}^{\mathrm{i}}(\mathrm{q}, \omega)=\int_{0}^{\mathrm{y}_{0}} \cos \left(\frac{\omega \mathrm{y}}{\mathrm{v}}\right) \mathrm{I}_{\mathrm{m}}(|\mathrm{q}| \mathrm{r}) \cos (\mathrm{m} \varphi) \mathrm{dy} \\
& \mathrm{S}_{\mathrm{m}}^{\mathrm{o}}(\mathrm{q}, \omega)=\int_{\mathrm{y}_{0}}^{\infty} \sin \left(\frac{\omega \mathrm{y}}{\mathrm{v}}\right) \mathrm{K}_{\mathrm{m}}(|\mathrm{q}| \mathrm{r}) \sin (\mathrm{m} \varphi) \mathrm{dy} \\
& \mathrm{S}_{\mathrm{m}}^{\mathrm{i}}(\mathrm{q}, \omega)=\int_{0}^{\mathrm{y}_{0}} \sin \left(\frac{\omega \mathrm{y}}{\mathrm{v}}\right) \mathrm{I}_{\mathrm{m}}(|\mathrm{q}| \mathrm{r}) \sin (\mathrm{m} \varphi) \mathrm{dy} \\
& \Gamma_{\mathrm{m}}(\mathrm{q}, \omega)=\int_{-\mathrm{y}_{0}}^{\mathrm{y}_{0}} \exp \left(-\frac{\mathrm{i} \omega \mathrm{y}}{\mathrm{v}}\right) \mathrm{I}_{\nu_{\mathrm{m}}(\omega)}\left[\left(\frac{\varepsilon_{\perp}(\omega)}{\varepsilon_{\|}(\omega)}\right)^{1 / 2}|\mathrm{q}| \mathrm{r}\right] \cos (\mathrm{m} \varphi) \mathrm{dy} \\
& \Sigma_{\mathrm{m}}(\mathrm{q}, \omega)=\int_{-\mathrm{y}_{0}}^{\mathrm{y}_{0}} \exp \left(-\frac{\mathrm{i} \omega \mathrm{y}}{\mathrm{v}}\right) \mathrm{I}_{\nu_{\mathrm{m}}(\omega)}\left[\left({\frac{\varepsilon_{\perp}(\omega)}{\varepsilon_{\|}(\omega)}}^{1 / 2}|\mathrm{q}| \mathrm{r}\right] \sin (\mathrm{m} \varphi) \mathrm{dy}\right.
\end{aligned}
$$

With the definitions, the surface plasmon excitation probability becomes 


$$
\begin{aligned}
\frac{\mathrm{dP}^{\text {surf }}(\omega)}{\mathrm{d} \omega}= & \frac{-\mathrm{e}^{2}}{4 \pi^{3} \varepsilon_{0} \hbar_{\mathrm{v}^{2}}} \sum_{\mathrm{m} \geqslant 0}\left(2-\delta_{0, \mathrm{~m}}\right) \\
& \times \int_{-\infty}^{\infty} \mathrm{dq}\left(4 \operatorname{Im}\left[\mathrm{A}^{\prime}(\mathrm{q}, \omega)\right]\left\{\left[\mathrm{C}_{\mathrm{m}}^{\mathrm{o}}(\mathrm{q}, \omega)\right]^{2}+\left[\mathrm{S}_{\mathrm{m}}^{\mathrm{o}}(\mathrm{q}, \omega)\right]^{2}\right\}\right. \\
& +4 \operatorname{Im}\left[\mathrm{C}^{\prime}(\mathrm{q}, \omega)\right]\left[\mathrm{C}_{\mathrm{m}}^{\mathrm{o}}(\mathrm{q}, \omega) \mathrm{C}_{\mathrm{m}}^{\mathrm{i}}(\mathrm{q}, \omega)+\mathrm{S}_{\mathrm{m}}^{\mathrm{o}}(\mathrm{q}, \omega) \mathrm{S}_{\mathrm{m}}^{\mathrm{i}}(\mathrm{q}, \omega)\right] \\
& +2 \operatorname{Im}\left\{\mathrm{B}_{\mathrm{m}}^{\prime}(\mathrm{q}, \omega)\left[\mathrm{C}_{\mathrm{m}}^{\mathrm{o}}(\mathrm{q}, \omega) \Gamma_{\mathrm{m}}(\mathrm{q}, \omega)+\mathrm{i} \mathrm{S}_{\mathrm{m}}^{\mathrm{o}}(\mathrm{q}, \omega) \Sigma_{\mathrm{m}}(\mathrm{q}, \omega)\right]\right\} \\
& \left.+2 \operatorname{Im}\left\{\mathrm{D}_{\mathrm{m}}^{\prime}(\mathrm{q}, \omega)\left[\mathrm{C}_{\mathrm{m}}^{\mathrm{i}}(\mathrm{q}, \omega) \Gamma_{\mathrm{m}}(\mathrm{q}, \omega)+\mathrm{i} \mathrm{S}_{\mathrm{m}}^{\mathrm{i}}(\mathrm{q}, \omega) \Sigma_{\mathrm{m}}(\mathrm{q}, \omega)\right]\right\}\right)
\end{aligned}
$$

where

$$
\begin{aligned}
& \mathrm{A}_{\mathrm{m}}^{\prime}(\mathrm{q}, \omega)=\frac{\mathrm{A}_{\mathrm{m}}(\mathrm{q}, \omega)}{\mathrm{K}_{\mathrm{m}}\left(|\mathrm{q}| \mathrm{r}_{0}\right)}, \\
& \mathrm{B}_{\mathrm{m}}^{\prime}(\mathrm{q}, \omega)=\frac{\mathrm{B}_{\mathrm{m}}(\mathrm{q}, \omega)}{\mathrm{K}_{\mathrm{m}}\left(|\mathrm{q}| \mathrm{r}_{0}\right)}, \\
& \mathrm{C}_{\mathrm{m}}^{\prime}(\mathrm{q}, \omega)=\frac{\mathrm{C}_{\mathrm{m}}(\mathrm{q}, \omega)}{\mathrm{I}_{\mathrm{m}}\left(|\mathrm{q}| \mathrm{r}_{0}\right)}
\end{aligned}
$$

and

$$
\mathrm{D}_{\mathrm{m}}^{\prime}(\mathrm{q}, \omega)=\frac{\mathrm{D}_{\mathrm{m}}(\mathrm{q}, \omega)}{\mathrm{I}_{\mathrm{m}}\left(|\mathrm{q}| \mathrm{r}_{0}\right)}
$$

\section{§ 5. DetERMination OF THE VOLUME PLASMON EXCITATION PROBability}

Based upon the local response assumption, the energy loss of an electron penetrating through a graphitic carbon cylinder can be calculated using equation (10). On each unit path length dy of the trajectory, the electron can be assumed to travel through a infinitely thin planar graphitic crystal with its $\mathrm{c}$ axis oriented along the radial unit vector of the cylindrical coordinates at the position $r_{0}$ (figures 2 and 5).
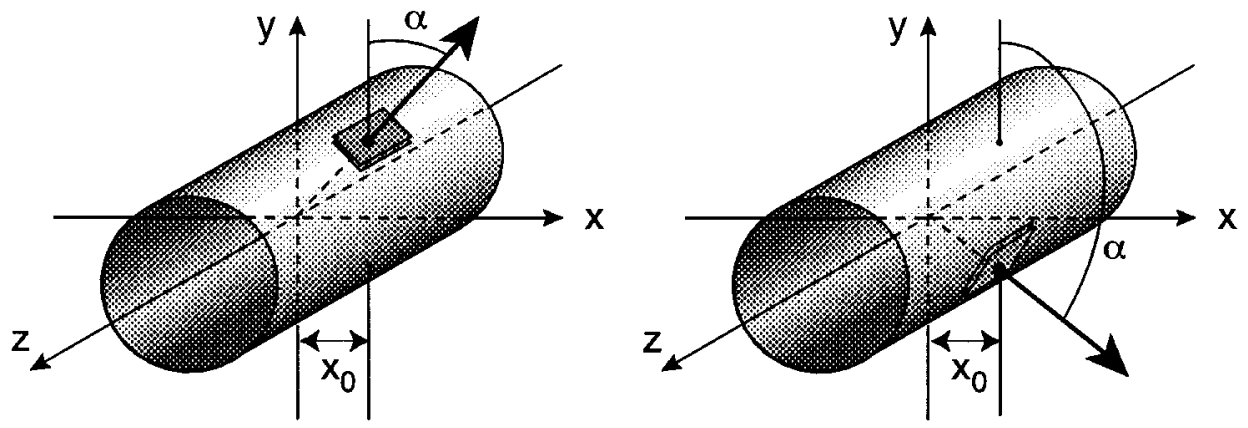

Figure 5. Model for the determination of the volume plasmon excitation probability of an electron penetrating a carbon nanotube. On the infinitesimal path interval $\mathrm{d} y$ the electron travels through a uniaxial graphitic layer with its $c$ axis oriented radially at the position of the electron. The figure shows the orientation of the graphitic layers for two different positions of the electron. 
The angle $\alpha$ between the $\mathrm{c}$ axis of the oriented piece of graphite and the trajectory of the electron depends on the position of the electron. If the position is parametrized by y (distance of the electron to the $(\mathrm{x}, \mathrm{z})$ plane), then

$$
\cos \alpha=\frac{\mathrm{y}}{\left(\mathrm{x}_{0}^{2}+\mathrm{y}^{2}\right)^{1 / 2}}
$$

and

$$
\sin \alpha=\frac{\mathrm{x}_{0}}{\left(\mathrm{x}_{0}^{2}+\mathrm{y}^{2}\right)^{1 / 2}} .
$$

The volume plasmon excitation probability of an electron penetrating a carbon nanotube can then be obtained from equation (10) by numerical integration over the trajectory of the electron:

$$
\frac{\mathrm{dP}^{\text {volume }}(\omega)}{\mathrm{d} \omega}=\int_{-\mathrm{y}_{0}}^{\mathrm{y}_{0}} \mathrm{dy} \frac{\mathrm{d}^{2} \mathrm{P}^{\text {volume }}(\omega, \mathrm{y})}{\mathrm{d} \omega \mathrm{dy}},
$$

where $\mathrm{y}_{0}$ is given as before by $\mathrm{y}_{0}=\left(\mathrm{a}^{2}-\mathrm{x}_{0}^{2}\right)^{1 / 2}$. With the explicit expression for the surface and volume plasmon excitation probabilities (equations (36) and (39)) the total plasmon excitation probability of a carbon nanotube is

$$
\frac{\mathrm{dP}^{\text {total }}(\omega)}{\mathrm{d} \omega}=\frac{\mathrm{dP}^{\text {volume }}(\omega)}{\mathrm{d} \omega}+\frac{\mathrm{dP}^{\text {surf ace }}(\omega)}{\mathrm{d} \omega}
$$

As mentioned in $\S 3$, the inner cavity of a carbon nanotube might be of importance for the volume plasmon excitation probability. In order not to overestimate this probability we propose to adapt the integration range for this case:

$$
\frac{\mathrm{dP}^{\text {volume }}(\omega)}{\mathrm{d} \omega}=\int_{-\mathrm{y}_{0}}^{-\mathrm{y}_{\mathrm{i}}} \mathrm{dy} \frac{\mathrm{d}^{2} \mathrm{P}^{\text {volume }}(\omega, \mathrm{y})}{\mathrm{d} \omega \mathrm{dy}}+\int_{\mathrm{y}_{\mathrm{i}}}^{\mathrm{y}_{0}} \mathrm{dy} \frac{\mathrm{d}^{2} \mathrm{P}^{\text {volume }}(\omega, \mathrm{y})}{\mathrm{d} \omega \mathrm{dy}} \text {. }
$$

$y_{i}=\left(r_{i}^{2}-x_{0}^{2}\right)^{1 / 2}$, where $r_{i}$ is the radius of the inner cavity, so that the integration only takes into account the filled part of the cylinder.

\section{§6. Conclusion}

In a recent publication (Stöckli et al. 1998a) it has been shown by means of the example of nested concentric-shell fullerenes that the inclusion of anisotropy in the non-relativistic local dielectric response theory for the excitation of the plasmons of carbon nanostructures introduces important changes compared with the isotropic model. Since then, experimental EELS data of multishell fullerenes have become available (Stöckli et al. 1998b). The comparison of those data with the simulations effectuated with the two formalisms (isotropic and anisotropic) shows that the inclusion of anisotropy in the model is essential in order to reproduce the data. The calculations presented in this contribution represent a straightforward continuation of the work on the theoretical background for the interpretation of plasmon loss electron-energy-loss spectra of graphitic carbon nanoparticles. In fact, the formalism developed here allows electron-energy-loss spectra of carbon nanotubes to be simulated as a function of their geometrical parameters for any impact parameter (electron passing inside and outside the particle) as well as intensity line profiles of energy-filtered images. It represents one of the missing pieces for better understanding of the physical properties of carbon nanostructures. We are confident that a 
detailed comparison of the experimental data of nested concentric-shell fullerenes and multiwall carbon nanotubes with simulations of the plasmon excitation probabilities based on our calculations will contribute to a better understanding of the physical properties of multiwall carbon nanostructures. In particular, it will be possible with our model to investigate how the intrinsic properties of multishell fullerenes differ from those of multiwall nanotubes and from those of planar graphite.

\section{ACKNOWLEDGEMENTS}

This work was partially financed by the Swiss National Science Foundation, under grant 2100-037660. Their support is gratefully acknowledged.

\section{A PPENDIX A}

From the first of the Maxwell inhomogeneous equations relating the displacement field to the charge density and the phenomenological relation between the displacement and the electric field the following equation for the electric field inside an infinitely large medium with the anisotropy of a carbon nanotube is found:

$$
\nabla_{\mathbf{r}} \cdot \tilde{\varepsilon}(\omega) \mathbf{E}\left(\mathbf{r}, \mathbf{r}_{0}\right)=\frac{\rho\left(\mathbf{r}, \mathbf{r}_{0}\right)}{\varepsilon_{0}} .
$$

The charge density represents the incoming probe electron and can be written in terms of a Dirac function:

$$
\rho\left(\mathbf{r}, \mathbf{r}_{0}\right)=-\mathrm{e} \delta\left(\mathbf{r}-\mathbf{r}_{0}\right)
$$

In order to solve this inhomogeneous equation we introduce the scalar function $\Phi\left(\mathbf{r}, \mathbf{r}_{0}\right)$ defined by

$$
\tilde{\varepsilon}(\omega) \mathbf{E}\left(\mathbf{r}, \mathbf{r}_{0}\right)=-\nabla_{\mathbf{r}} \Phi\left(\mathbf{r}, \mathbf{r}_{0}\right) .
$$

Equation (A 1) then becomes

$$
\nabla_{\mathrm{r}}^{2} \Phi\left(\mathbf{r}, \mathbf{r}_{0}\right)=\frac{\mathrm{e}}{\varepsilon_{0}} \delta\left(\mathbf{r}, \mathbf{r}_{0}\right)
$$

Formally this equation is identical with the Laplace equation. However, $\Phi\left(\mathbf{r}, \mathbf{r}_{0}\right)$ is not the Coulomb potential, but only a mathematical construction introduced for convenience. From equation (A 4$)$ it is found that $\Phi\left(\mathbf{r}, \mathbf{r}_{0}\right)$ is given by

$$
\begin{aligned}
\Phi\left(\mathbf{r}, \mathbf{r}_{0}\right) & =-\frac{\mathrm{e}}{4 \pi \varepsilon_{0}\left|\mathbf{r}-\mathbf{r}_{0}\right|} \\
& =-\frac{\mathrm{e}}{2 \pi \varepsilon_{0}} \sum_{\mathrm{m} \geqslant 0}\left(2-\delta_{0, \mathrm{~m}}\right) \cos \left[\mathrm{m}\left(\varphi-\varphi_{0}\right)\right] \int_{-\infty}^{\infty} \frac{\mathrm{dq}}{2 \pi} \exp (\text { iqz }) \mathrm{L}_{\mathrm{m}}\left(|\mathrm{q}| \mathrm{r},|\mathrm{q}| \mathrm{r}_{0}\right),
\end{aligned}
$$

where the function $\mathrm{L}_{\mathrm{m}}\left(|\mathrm{q}| \mathrm{r},|\mathrm{q}| \mathrm{r}_{0}\right)$ is given in equation $(18 b)$. Using the definition of the function $\Phi\left(\mathbf{r}, \mathbf{r}_{0}\right)$ and the gradient in cylindrical coordinates, the electric field is obtained from equation (A 5): 


$$
\begin{aligned}
\mathbf{E}\left(\mathbf{r}, \mathbf{r}_{0}\right)= & -\frac{\mathrm{e}}{2 \pi \varepsilon_{0}} \sum_{\mathrm{m} \geqslant 0}\left(2-\delta_{0, \mathrm{~m}}\right) \int_{-\infty}^{\infty} \frac{\mathrm{dq}}{2 \pi} \\
& \times\left(\begin{array}{l}
\frac{1}{\varepsilon_{\|}(\omega)} \exp (\mathrm{iqz}) \cos \left[\mathrm{m}\left(\varphi-\varphi_{0}\right)\right] \frac{\partial}{\partial \mathrm{r}} \mathrm{L}_{\mathrm{m}}\left(|\mathrm{q}| \mathrm{r},|\mathrm{q}| \mathrm{r}_{0}\right) \\
-\frac{1}{\varepsilon_{\perp}(\omega)} \exp (\mathrm{iqz}) \frac{\mathrm{m} \sin \left[\mathrm{m}\left(\varphi-\varphi_{0}\right)\right]}{\mathrm{r}} \mathrm{L}_{\mathrm{m}}\left(|\mathrm{q}| \mathrm{r},|\mathrm{q}| \mathrm{r}_{0}\right) \\
\frac{1}{\varepsilon_{\perp}(\omega)} \mathrm{iq} \exp (\mathrm{iqz}) \cos \left[\mathrm{m}\left(\varphi-\varphi_{0}\right)\right] \mathrm{L}_{\mathrm{m}}\left(|\mathrm{q}| \mathrm{r},|\mathrm{q}| \mathrm{r}_{0}\right.
\end{array}\right)
\end{aligned}
$$

\section{REFERENCES}

Ajayan, P. M., Ijjima, S., and Ichinashi, T., 1992, Phys. Rev. B, 47, 6859.

Bachtold, A., Henry, M., Terrier, C., Strunk, C., Schönenberger, C., Salvetat, J.-P., Bonard, J.-M., and Forró, L., 1998, Appl. Phys. Lett., 73, 274.

Bausells, J., Rivacoba, A., and Echenique, P. M., 1987, Surf. Sci., 190, 1015.

Bez ryadin, A., Verschueren, A. R. M., Tans, S. J., and Dekker, C., 1998, Phys. Rev. Lett., 80, 4036.

Bonard, J.-M., Stöckli, T., de Heer, W. A., Châtelain, A., Charlier, J.-C., Blase, X., De Vita, A., Car, R., Salvetat, J.-P., and Forró, L., 1997a, Phys. Rev. Lett., (submitted).

Bonard, J.-M., Stora, T., Salvetat, J.-P., Maier, F., Stöckli, T., Duschl, K., Forró, L, De Heer, W., and Châtelan, A., 1997b, Adv. Mater., 9, 827.

Bursill, L. A., Stadelmann, P. A., Peng, J. L., and Prawer, S., 1992, Phys. Rev. B, 49, 2882.

Carroll, D. L., Redlich, P., Ajayan, P. M., Charlier, J. C., Blase, X., De Vito, A., and CAR, R., 1997, Phys. Rev. Lett., 78, 2811.

Chu, Y. T., Warmack, R. J., Ritchie, R. H., Luttle, J. W., Becker, R. S., and Ferrell, T. L., 1984, Particle Acceleration, 16, 13.

Daniels, J., Festenberg, C., Raether, H., and Zeppenfeld, K., 1979, Optical Constants of Solids by Electron Spectroscopy (Berlin: Springer Verlag).

de Heer, W. A., Châtelain, A., and Ugarte, D., 1995, Science, 270, 1179.

Dujardin, E., Ebbesen, T. W., Krishnan, A., and Treacy, M. M. J., 1998, Adv. Mater., 10, 611 .

Ebbesen, T. W., and Ajayan, P. M., 1992, Nature, 358, 220.

Echenique, M., Howie, A., and Wheatley, D. J., 1987, Phil. Mag. B, 56, 335.

Egerton, R. F., 1996, Electron Energy-Loss Spectroscopy in the Electron Microscope (New York: Plenum Press).

Ferrell, T. L, Anderson, V. E., Echenique, P. M., and Warmack, R. J., Phys. Rev. B, 35, 7365.

Ferrell, 'T. L., and Echenique, P. M., 1985, Phys. Rev. Lett., 55, 1562.

Frank, S., Poncharal, P., Wang, Z. L., and De Heer, W. A., 1998, Science, 280, 1744.

Hamada, N., Sawada, S., and Oshiyama, A., 1992, Phys. Rev. Lett., 68, 1579.

Hubbard, J., 1955a, Proc. Phys. Soc. A, 68, 976; 1955b, ibid. 68, 441.

IjJiмa, S., 1991, Nature, 354, 56.

Jackson, J. D., 1975, Classical Electrodynamics (New York: Wiley).

Journet, C., Maser, W. K., Bernier, P., Loiseau, A., Lamy de la Chapelle, M., Lefrant, S., Deniard, P., Lee, R., and Fischer, J. E., 1997, Nature, 388, 756.

Krätschmer, W., Lami, L. D., Fostiropoulos, K., and Huffman, D. R., 1990, Nature, 347,354 .

Kroto, H. W., Health, J. R., O’Brian, S. C., Curl, R. F., and Smalley, R. E., 1985, Nature, 381, 162.

Kuzuo, R., Terauchi, M., and Tanaka, M., 1992, Jap. J. appl. Phys., Pt 2, 31, L1484.

Lucas, A. A., Henrad, L., and Lambin, P., 1994, Phys. Rev. B, 49, 2888; 1995, Nuclear Instruments Meth. B, 96, 465.

Mintmire, J. W., Dunlap, B. I., and White, C. T., 1992, Phys. Rev. Lett., 68, 631. 
Ritchie, R. H., 1957, Phys. Rev., 106, 874.

Rivacoba, A., Apell, P., and Zabala, N., 1995, Nucl. Instrum. Meth. B, 96, 470.

Saito, R., Fujita, G., Dresselhaus, G., and Dresselhaus, M. S., 1992, Appl. Phys. Lett., 60, 2204.

Salvetat, J.-P., Kulik, A. J., Briggs, G. A. D., Bonard, J.-M., Stöckli, T., Burnham, N., and Forró, L., 1999, Adv. mater., 11, 161.

Stéphan, O., Ajayan, P. M., Colliex, C., Cyrot-Lackmann, F., and Sandré, E., 1996, Phys. Rev. B, 53, 13824.

Stöckli, T., Bonard, J.-M., Châtelain, A., Wang, Z. L., and Stadelmann, P., 1998a, Phys. Rev. B, 57, 15599.

Stöckli, T., Bonard, J.-M., Stadelmann, P. A., and Châtelain, A., 1997a, Z. Phys. D, 40, 425.

Stöckli, T., Stadelmann, P. A., and Châtelan, A., 1997b, Microsc. Microanal. Microstruct., 8, 145.

Stöckli, T., Wang, Z. L., Bonard, J.-M., Stadelmann, P., and Châtelain, A., 1998b, Proceedings of 12th International Winterschool of the Electronic Properties of Novel Materials, edited by H. Kuzmany, J. Fink, M. Mehring and S, Roth. (New York: American Institute of Physics). pp. 439-442.

Tans, S. J., Devoret, M. H., Dai, H., Thess, A., Smalley, R. E., Geerligs, L. J., and DekKer, C., 1997, Nature, 386, 474.

Thess, A., Lee, R., Nikolaev, P., Dai, H., Petit, P., Robert, J., Xu, C., Lee, Y. H., Kim, S. G., Colbert, D. T., Scuseria, G., Tomanek, T., Fischer, J. E., and Smalley, R. E., 1996, Science, 273, 483.

Tosatti, E., 1969, Nuovo Cim. B, 63, 54.

Ugarte, D., 1992, Nature, 359, 707.

Ugarte, D., Colliex, C., and Trebbia, P., 1992, Phys. Rev. B, 45, 4332.

Walsh, C. A., 1991, Phil. Mag. B, 63, 1063.

Wang, Z. L., 1996, Micron, 27, 265.

Wang, Z. L., and Cowley, J. M., 1987, Ultramicroscopy, 21, 77.

Watson, G. N., 1996, A Treatise on the Theory of Bessel Functions (Cambridge University Press).

Wessjohann, H. G., 1974, Z. Phys., 269, 269.

Yase, K., Horiuchi, S., Kyotani, M., Yumura, M., Uchida, K., Ohshima, S., Kuriki, Y., Ikazaki, F., and Yamahira, N., 1996, Thin Solid Films, 273, 222.

Zabala, N., and Rivacoba, A., 1991, Ultramicroscropy, 35, 145.

Zabala, N., Rivacoba, A., and Echenique, P. M., 1989, Surf. Sci., 209, 465. 